\title{
The Subthalamic Nucleus Is One of Multiple Innervation Sites for Long-Range Corticofugal Axons: A Single-Axon Tracing Study in the Rat
}

\author{
Takako Kita and Hitoshi Kita \\ Department of Anatomy and Neurobiology, College of Medicine, The University of Tennessee Health Science Center, Memphis, Tennessee 38163
}

The frontal cortex provides strong excitatory inputs to the subthalamic nucleus (STN), and these cortico-STN inputs play critical roles in the control of basal ganglia activity. It has been assumed from anatomical and physiological studies that STN is innervated mainly by collaterals of thick and fast conducting pyramidal tract axons originating from the frontal cortex deep layer $V$ neurons, implying that STN directly receives efferent copies of motor commands. To more closely examine this assumption, we performed biotinylated dextran amine anterograde tracing studies in rats to examine the cortical layer of origin, the sizes of parent axons, and whether or not the cortical axons emit any other collaterals to brain areas other than STN. This study revealed that the cortico-STN projection is formed mostly by collaterals of a small fraction of small-to-medium-sized long-range corticofugal axons, which also emit collaterals that innervate multiple other brain sites including the striatum, associative thalamic nuclei, superior colliculus, zona incerta, pontine nucleus, multiple other brainstem areas, and the spinal cord. The results imply that some layer $\mathrm{V}$ neurons are involved in associative control of movement through multiple brain innervation sites and that the cortico-STN projection is one part of this multiple corticofugal system.

\section{Introduction}

The frontal cortex provides strong excitatory inputs to the subthalamic nucleus (STN), and these cortico-STN inputs play critical roles in the control of basal ganglia activity. Physiological, focal lesion, and deep brain stimulation (DBS) studies suggest that the cortico-STN inputs may also play critical roles in the development of abnormal activity in the basal ganglia that lead to motor and cognitive dysfunctions such as Parkinson's disease (Albin et al., 1989; DeLong, 1990; Graybiel et al., 1994; Hornykiewicz, 2006; Israel and Bergman, 2008).

Retrograde tracing studies have suggested that the frontal cortex, including the motor and prefrontal cortices, innervate STN (Künzle, 1978; Monakow et al., 1979; Canteras et al., 1990; Orieux et al., 2002). Electrical stimulation of the motor cortex evokes a short latency excitation in STN (Kitai and Deniau, 1981; Fujimoto and Kita, 1993; Kita, 1994; Nambu et al., 2000). STN stimulation evoked short latency antidromic spikes in the motor cortex layer V neurons (Paz et al., 2005). Stimulation of the cat medullary pyramid evoked a short-latency excitation in STN, which was considered to be due to antidromic activation of parent axons that emit STN collaterals (Giuffrida et al., 1985). A

\footnotetext{
Received Nov. 14, 2011; revised Feb. 2, 2012; accepted March 12, 2012.

Author contributions: H.K. designed research; T.K. and H.K. performed research; T.K. and H.K. analyzed data; T.K. and H.K. wrote the paper.

This work was supported by the National Institute of Neurological Disorders and Stroke Grants NS47085 and NS-57236. We thank J. Butler for technical assistance and R. Kita for editing the manuscript.

Correspondence should be addressed to Hitoshi Kita, Department of Anatomy and Neurobiology, College of Medicine, The University of Tennessee Health Science Center, 855 Monroe Avenue, Memphis, TN 38163. E-mail: hkita@uthsc.edu.

DOI:10.1523/JNEUROSCI.5717-11.2012

Copyright $\odot 2012$ the authors $\quad 0270-6474 / 12 / 325990-10 \$ 15.00 / 0$
}

Golgi study described that descending axons of various sizes in the cerebral peduncle emit collaterals innervating STN (Iwahori, 1978). Based on these observations, we have assumed that cortico-STN innervations are formed mainly by collaterals of thick, fast-conducting pyramidal tract axons originating from the frontal cortex deep layer V neurons (Kitai and Kita, 1987; Kita, 1994), implying that STN directly receives efferent copies of motor commands. However, the true cortical layer of origin of the axons, the size of the axons, and whether or not the axons emit collaterals to any other brain areas other than STN are as yet unknown. These questions are important not only for the understanding of the functional roles of the cortico-STN innervations in normal conditions but also for the understanding of the effects of STN-DBS in movement disorders, which most likely activates neurons and axons, including the afferent axons, at and near the stimulus site.

To investigate morphological details of cortical neurons innervating STN, we traced single axons labeled with small injections of biotinylated dextran amine (BDA) in the motor cortex of rats. This study revealed that the cortico-STN projection is formed mostly by collaterals of a small fraction of small to medium sized long-range corticofugal axons that also emit collaterals that innervate multiple other brain sites, including the striatum (Str), thalamus, lower brainstem, and spinal cord.

\section{Materials and Methods}

The experiments were performed on adult male Sprague Dawley rats (270-320 g, Charles River Laboratories) in compliance with the National Institutes of Health Guide for Care and Use of Laboratory Animals and the University of Tennessee Health Science Center Guide for the Use and Care of Laboratory Animals in Research. We used the BDA (BDA-10000, Invitrogen) anterograde tracing method to label the STN innervating axons 
that originated from the motor cortex. Rats were anesthetized with an intraperitoneal injection of a mixture of ketamine $(60 \mathrm{mg} / \mathrm{kg}$, Pfizer $)$ and xylazine (10 mg/kg, Agri-lab) and placed on a stereotaxic apparatus. BDA injections were targeted to the rostral portions of the lateral and medial agranular cortices (AGl and AGm, respectively), which provide dense projections to STN (Canteras et al., 1990; Degos et al., 2008). The injection coordinates in AGl were anterior $(\mathrm{A})=3.3-3.5 \mathrm{~mm}$ from the bregma and lateral $(\mathrm{L})=2.8-3.2 \mathrm{~mm}$ from the midline; in $\mathrm{AGm}, \mathrm{A}=$ $4.3-4.7 \mathrm{~mm}$ and $\mathrm{L}=1.2-1.6 \mathrm{~mm}$. All injections were into the left cortex. Craniotomies over these areas were performed, and the tips of the BDAcontaining glass micropipettes (3\% dissolved in saline, tip diameter of 5-10 $\mu \mathrm{m}$ ) were placed $1-1.2 \mathrm{~mm}$ below the cortical surface. BDA was injected iontophoretically by $100 \mathrm{~ms}$ on/off, with $100 \mathrm{nA}$ positive current pulses for $10 \mathrm{~min}$.

To compare the somatic locations and sizes of cortico-STN neurons to those of pyramidal tract neurons, Fluorogold (FG; Fluorochrome) was injected into the pyramidal decussation of two rats. For the FG injections, glass micropipettes (tip diameters of $\sim 10 \mu \mathrm{m}$ ) glued to the needle of a 10 $\mu$ l Hamilton microsyringe were filled with $0.1 \%$ FG in saline. The rats were anesthetized, and the cisterna magna was opened for the insertion of the pipette. FG $(0.3 \mu \mathrm{l})$ was injected slowly $(0.05 \mu \mathrm{l} / \mathrm{min})$ by pushing the microsyringe plunger with an electric actuator.

After $14-18 \mathrm{~d}$ of survival for BDA and $10 \mathrm{~d}$ for FG injections, the rats were deeply anesthetized with a mixture of ketamine $(100 \mathrm{mg} / \mathrm{kg})$ and xylazine $(20 \mathrm{mg} / \mathrm{kg})$ and were perfused through the heart with $10-20 \mathrm{ml}$ of isotonic saline followed by a fixative. The fixative was a mixture of $4 \%$ formaldehyde and $0.2 \%$ picric acid in a $0.12 \mathrm{M}$ sodium phosphate buffer (200-300 ml, pH 7.4). After perfusion, the brains were removed and postfixed overnight at $4{ }^{\circ} \mathrm{C}$, and then equilibrated in a $10 \%$ followed by a $30 \%$ sucrose phosphate buffer, $\mathrm{pH}$ 7.4. The brains were then cut into 40 $\mu \mathrm{m}$ sections on a freezing microtome (sagittal for BDA, coronal for FG).

For the visualization of BDA, the sections were rinsed with $\mathrm{PBS}, \mathrm{pH}$ 7.6 , and then incubated in PBS containing $2 \%$ avidin-biotin-peroxidase complex (ABC; 1:100, Vector Laboratories) and 0.2\% Triton X-100 for $12 \mathrm{~h}$. After rinsing several times with PBS, the sections were incubated in PBS containing 3, $3^{\prime}$-diaminobenzidine (DAB) (0.05\%), $\mathrm{NiCl}(0.001 \%)$, and $\mathrm{H}_{2} \mathrm{O}_{2}(0.003 \%)$. This process stained the BDA-labeled axons a dark blue color. The wet sections were examined under a microscope. We discarded sections of seven rats because the BDA injections were too faint, and we were unable to find any labeled axons in the cerebral peduncle. We accumulated $10 \mathrm{AGl}$ and $10 \mathrm{AGm}$ injections with successful labeling of layer $\mathrm{V}$ neurons with 7-21 labeled axons in the cerebral peduncle ventral to the STN. Sections containing BDA-labeled neurons at the injection sites were further processed for immunostaining for neuron-specific nuclear protein (NeuN). The NeuN monoclonal antibody (MAB377, Millipore) recognizes NeuN in most CNSs of vertebrates (information provided by Millipore). The sections were incubated overnight in PBS containing anti-NeuN (1:40,000), followed by biotinylated anti-mouse antibody $(0.5 \mu \mathrm{g} / \mathrm{ml}$; BA-2000, Vector Laboratories) for $1.5 \mathrm{~h}$ and finally in $\mathrm{ABC}$ for $1.5 \mathrm{~h}$. The peroxidase was then visualized by incubating the sections in PBS containing 3,3'-diaminobenzidine $(0.05 \%)$ and $\mathrm{H}_{2} \mathrm{O}_{2}(0.003 \%)$. The immunostaining for $\mathrm{NeuN}$ stained the nuclei neurons a yellow-brown color and stained the cytoplasm a light yellow color. After several rinses, immunostained sections were mounted on gelatin-coated slides, air dried, dehydrated in graded alcohols to xylene, and coverslipped.

Immunohistochemistry for FG. The sections for immunohistochemistry for FG were preincubated in PBS containing 0.5\% nonfat dry milk and $0.2 \%$ Triton-X for $2-3 \mathrm{~h}$, and then incubated overnight in PBS containing rabbit anti-FG serum (1:15,000; a gift from Dr. H. T. Chang, Michigan State University, East Lansing, MI) (Chang et al., 1990). The sections were rinsed four times with PBS and incubated in biotinylated antirabbit antibody $(0.5 \mu \mathrm{g} / \mathrm{ml}$; BA-1000, Vector Laboratories) for $1.5 \mathrm{~h}$. Next, the sections were rinsed four times with PBS and incubated in ABC for $1.5 \mathrm{~h}$. Finally, the sections were rinsed four more times and then incubated in PBS containing $\mathrm{DAB}(0.05 \%), \mathrm{NiCl}(0.001 \%)$, and $\mathrm{H}_{2} \mathrm{O}_{2}$ $(0.003 \%)$. This reaction resulted in dark blue granular reaction products in the cytoplasm of FG-labeled neurons with virtually no nonspecific background staining.
To identify cortical layers, some of the sections stained for FG were further processed for immunostaining for NeuN or type 2 vesicular glutamate transporter (VGLUT2) (1:10,000; AB2251, Millipore Bioscience Research Reagents). VGLUT2 is rich in layer I and lower layer II/III of the agranular cortex. Also, layer V can be divided into an upper VGLUT2poor and a lower VGLUT2-rich sublayer, reflecting the differences in thalamic input density (Kubota et al., 2007). The former is referred to as layer $\mathrm{Va}$ and the latter as layer $\mathrm{Vb}$ (Morishima et al., 2011). The immunostaining procedures were very similar to those described for NeuN above. To estimate somatic size, some AGl and AGm neurons doubly labeled for FG and NeuN and with no obvious cuts to the somata were drawn using a $100 \times$ oil-immersion objective.

Axon tracing. BDA-labeled axons and neurons were drawn using a light microscope (BX50, Olympus) with $40 \times$ dry and $60 \times$ waterimmersion objectives and a camera lucida. We reconstructed only the neurons innervating STN in this study. The reconstructions began with thin axons bearing spherical varicosities in STN (called boutons hereafter to distinguish these from other irregularly shaped varicosities), followed by collateral axons innervating STN (called STN collaterals hereafter), and then with the parent axons to both rostral and caudal directions. Other collateral axons that were emitted from the parent axons and innervating other brain areas were also drawn. The reconstructions of most of the collateral axons, including STN collaterals, were often incomplete because they faded out and/or were inseparable from axons belonging to other neurons. Thus, it was not possible to accurately measure the sizes of the axonal arbors and the numbers of boutons. Microscopic images were acquired by a digital camera (D70, Nikon) and processed using a Macintosh computer with Photoshop (Adobe) and Canvas (Deneva).

\section{Results}

\section{Parent axons in the cerebral peduncle}

The BDA injections were targeted to label a small number of corticofugal axons per rat. This report is based on 10 each of AGland AGm-injected rats with successful labeling of layer $\mathrm{V}$ cells with labeling of 7-21 axons in the cerebral peduncle ventral to the STN. Eight of the 10 AGl-injected rats yielded one to three axons per rat bearing STN collaterals, while the remaining two rats had labeled axons in the cerebral peduncle but without STN collaterals. There were a total of 168 labeled axons in the cerebral peduncle of the 10 AGl-injected rats, of which 18 (10.7\%) had STN collaterals. Of the $10 \mathrm{AGm}$-injected rats, 5 rats had one or two cerebral peduncle axons each with STN collaterals, while the other 5 rats had labeled axons without STN collaterals. Of a total of 139 cerebral peduncle axons originating from AGm, 7 (5\%) emitted STN collaterals. The results below are based on a total of 25, $18 \mathrm{AGl}$ and $7 \mathrm{AGm}$, cortical axons with STN collaterals.

\section{STN collaterals}

The collateral axons in STN were very thin and bifurcated into long secondary or higher-order branches that took tortuous courses and bore sparse en passant boutons at various intervals. Pedunculated boutons (boutons at the tip of short stalks) were also occasionally observed on the axons (Fig. $1 A, C$, arrowheads). AGl neurons formed terminal fields in the rostrodorsal two thirds and the mid-mediolateral two thirds of STN. The terminals formed by axons of AGm neurons were found in a slightly larger area that covered slightly more caudal and medial areas than that of the AGl terminal field. The narrow most lateral and caudomedial portions of STN were free of innervation (Figs. 2, 3). The AGl and AGm projection sites greatly overlapped and coincided with the motor territory of STN (Canteras et al., 1990; Kolomiets et al., 2001). In 16 of 25 cases, the thin branches of axons innervating STN traveled in the dorsocaudal direction to the zona incerta (ZI); some of these emitted several branches with boutons en 
passant in ZI, while others faded out before reaching terminal areas (Figs. $1 B, 2$, 3 ). We found $4-41$ boutons (mean $=$ 23.2 boutons) on 1-11 bouton-bearing branches on each STN collateral of AGl neurons. We found higher numbers of 7-94 boutons (mean $=49.2$ boutons) on 1-13 bouton-bearing branches on STN collaterals of AGm neurons. The collaterals having only one or two branches belonged to those innervating more to ZI (Fig. 2A).

The collaterals innervating STN most often branched from the parent axons in the internal capsule at a level rostral to or in the cerebral peduncle immediately ventral to STN. In one neuron, a thin collateral that branched in the Str innervated STN. Figure $4, A$ and $B$, shows photomicrographs of parent axons with and without STN collaterals, respectively. Figure $4 C$ compares the diameters of these axons in the cerebral peduncle at the level of STN. To estimate diameters, each axon was observed using a $100 \times$ oil-immersion objective and assigned to one of $0.2-\mu \mathrm{m}$ increment categories by comparing its thin, smooth portion (between varicosities) to a calibrated scale projected through the drawing tube. Although precise diameter measurement was not possible because of the various degrees of axonal varicosity and staining intensity, it could be seen that STN collaterals arise from thin-to-medium-sized axons but not from thick axons.

\section{Destination of parent axons}

Table 1 summarizes the results of tracing axons of $18 \mathrm{AGl}$ and 7 AGm neurons. Sixteen of the 18 AGl-STN neurons were pyramidal tract neurons, of which 8 parent axons were traced to the pyramidal decussation or to the dorsal corticospinal tract, while the other 8 axons were traced to the medullary pyramid and then faded out. The parent axons of two nonpyramidal tract neurons were traced to the superior colliculus (SC) and to the pontine nucleus (Pn). All of the AGm-STN neurons were pyramidal tract neurons, and, therefore, most of the cortico-STN neurons from the agranular cortex were assumed to be pyramidal tract neurons.

\section{Somatic location and size}

All of the parent axons were traced back to the cortex, of which 12 of 18 axons in AGl and 5 of 7 axons in AGm, for a total of 17 axons, were traced to their originating soma. The remaining axons could not be traced back to the soma because the axons entered the BDA injection area with numerous labeled cell bodies and processes. Most of the identified somata were located in the middle to superficial part of layer V (Fig. 5). The somatic areas (i.e., areas within the outline of somata) of the $17 \mathrm{STN}$-projecting neurons ranged from 584 to $934 \mu \mathrm{m}^{2}$ with a mean of $758 \pm 112$ $\mu \mathrm{m}^{2}$. The neurons had slender apical dendrites that were largely
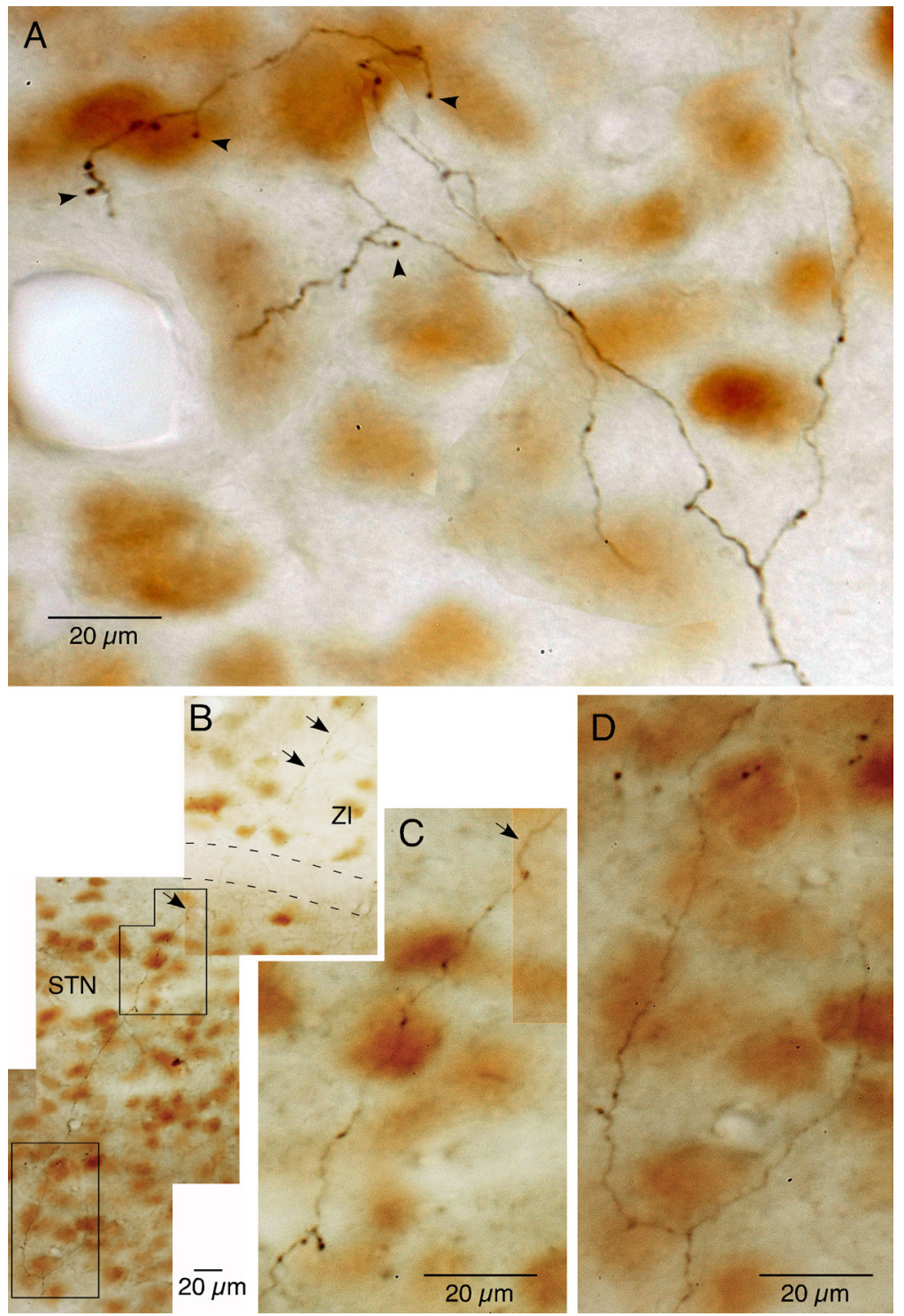

Figure 1. Photomontages of cortico-STN axons and boutons in NeuN-immunostained sections. $\boldsymbol{A}$, The collateral axons in STN One of the very thin branches traverses the white matter located dorsal to STN and enters ZI (marked by arrows). Areas marked in $\boldsymbol{B}$ are shown in higher magnification in $\boldsymbol{C}$ and $\boldsymbol{D}$.

spine free. The parent axons of STN collaterals were never traced to neurons with spine-rich dendrites in the injection sites.

To compare the somatic locations and sizes of STN-projecting neurons to other pyramidal tract neurons, FG was injected into the pyramidal decussation (Fig. 6A). A large number of neurons with various diameters were labeled in AGl and AGm. The somatic areas of labeled neurons ranged from 465 to $1100 \mu \mathrm{m}^{2}$ with a mean of $710 \pm 105 \mu \mathrm{m}^{2}(n=250)$. The sampled neurons included most of the labeled neurons having complete somata in $\mathrm{AGl}$ and AGm of the section shown in the Figure $6 \mathrm{~B}$. The somatic areas were not statistically different from those of STN-projecting neurons ( $p=0.35$, Mann-Whitney $U$ test), although Figure $6 E$ suggests that only medium-to-large neurons project to STN. The layer V can be separated into VGLUT2-poor sublayer Va and VGLUT2-rich sublayer Vb (Morishima et al., 2011). The AGl has a very thin $\mathrm{Va}$ and thick $\mathrm{Vb}$, while the $\mathrm{AGm}$ and granular cortex have $\mathrm{Va}$ and $\mathrm{Vb}$ with similar thicknesses. Most of the FG-labeled 


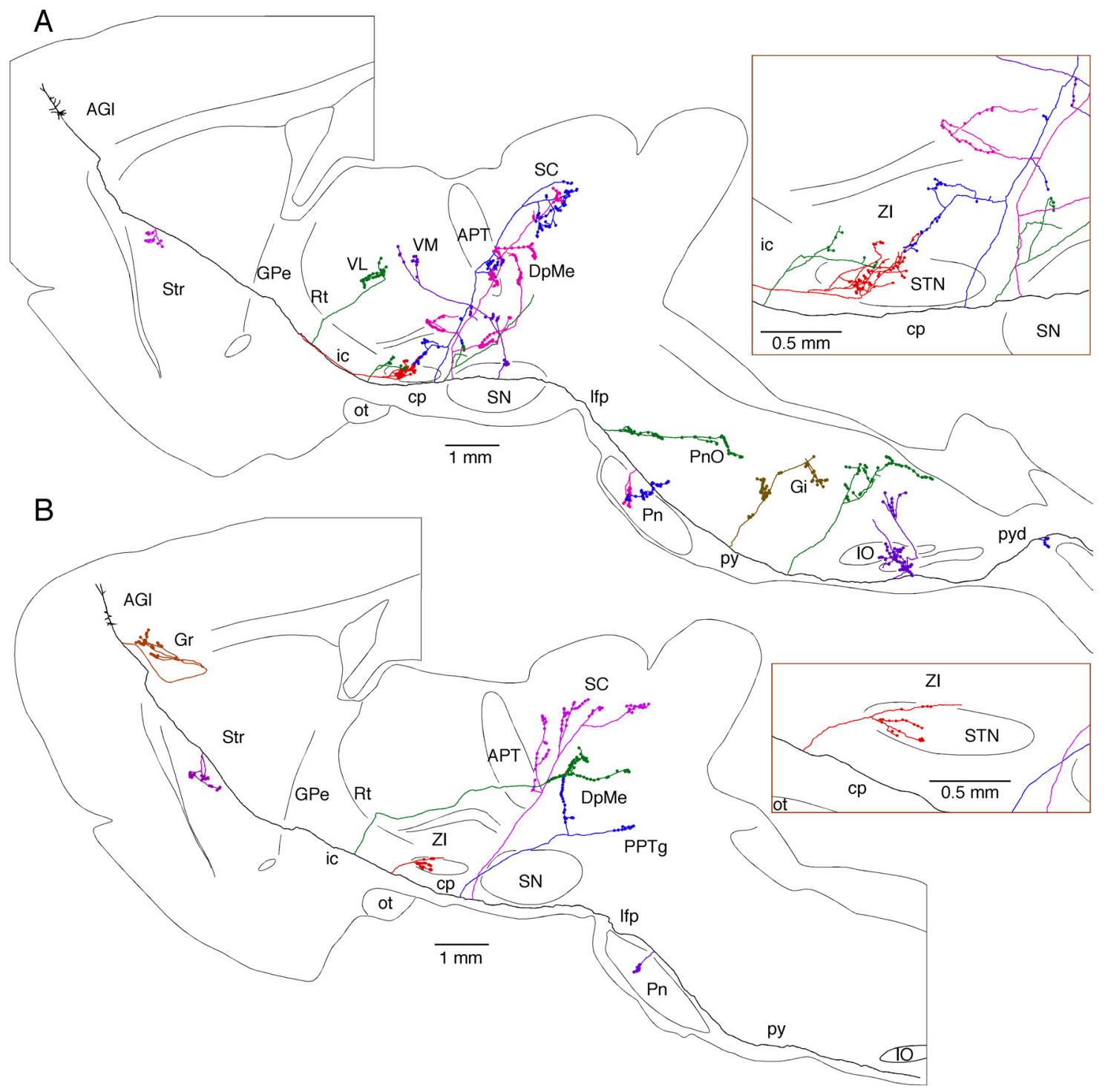

Figure 2. Axons of two AGl pyramidal tract neurons that emit multiple collaterals (shown with different colors) including STN collaterals. No cortical collateral was found on the neuron shown in $\boldsymbol{A}$, though this neuron had multiple collaterals innervating to Str, thalamic, mesencephalic, pontine, and medullary nuclei. The neuron shown in $\boldsymbol{B}$ had a cortical collateral innervating Gr and collaterals innervating Str, and mesencephalic and pontine nuclei. The STN collaterals of both neurons had thin branches entering ZI. One of the cerebral peduncle collaterals of neuron A emitted ZI branch-forming boutons. GPe, Globus pallidus external segment; Gr, granular cortex; ic, internal capsule; I0, inferior olive; Gi, gigantocellular reticular nucleus; py, medullary pyramid; pyd, pyramidal decussation; Rt, reticular thalamic nucleus; VM, ventromedial thalamic nucleus; ot, optic tract; $\mathrm{PnO}$, pontine reticular nucleus, oral part; $\mathrm{cp}$, cerebral peduncle; Ifp, Iongitudinal fasciculus of the pons.

neurons in AGl and AGm lie in layer $\mathrm{Vb}$ with no apparent depthdependent distribution of small and large neurons (Fig. 6D). These results and the somatic locations of STN-projecting neurons described above indicate that cortico-STN projections arise mainly from the superficial part of layer $\mathrm{Vb}$ of agranular cortices.

\section{Other innervation sites of cortico-STN neurons}

This study revealed that cortico-STN neurons emit collaterals that innervate several other brain sites, as summarized in Table 1. The fraction of neurons emitting collaterals in each brain site showed that overall distributions of the projection sites of AGl and AGm neurons were very similar, and therefore all of the neurons were pooled for the following discussions.

\section{Cortical collaterals}

Of the 17 parent axons traced to their somata, local cortical collaterals were found on 9 axons. Some of the collaterals innervating within or near the BDA injection sites were not traceable.
Some of the collaterals traced outside of the injection area traveled caudally and slightly medially to innervate to layers I-III of agranular cortex 1-4 mm away from the dendritic domain of the parent cell, and others traveled caudolaterally to layers I-III of the granular cortex (Figs. 2, 3). We did not find any cortical collateral in 8 of the 17 parent axons traced to their somata. There is a tendency for neurons that have cortical collaterals to also have Str collaterals ( 8 of 12 neurons) (Table 1$)$. No other clear morphological differences were found between the neurons with and without cortical collaterals.

\section{Striatal collaterals}

Thirteen of 25 parent axons emitted one or two collaterals in Str. Some of the collaterals were traceable for only short distances, while some others were traced to a large area extending $>1 \mathrm{~mm}^{3}$. There was a tendency for neurons that had cortical collaterals to also have Str collaterals, as described above. Also, similar to STN, we found larger terminal fields with more boutons on AGm ax- 


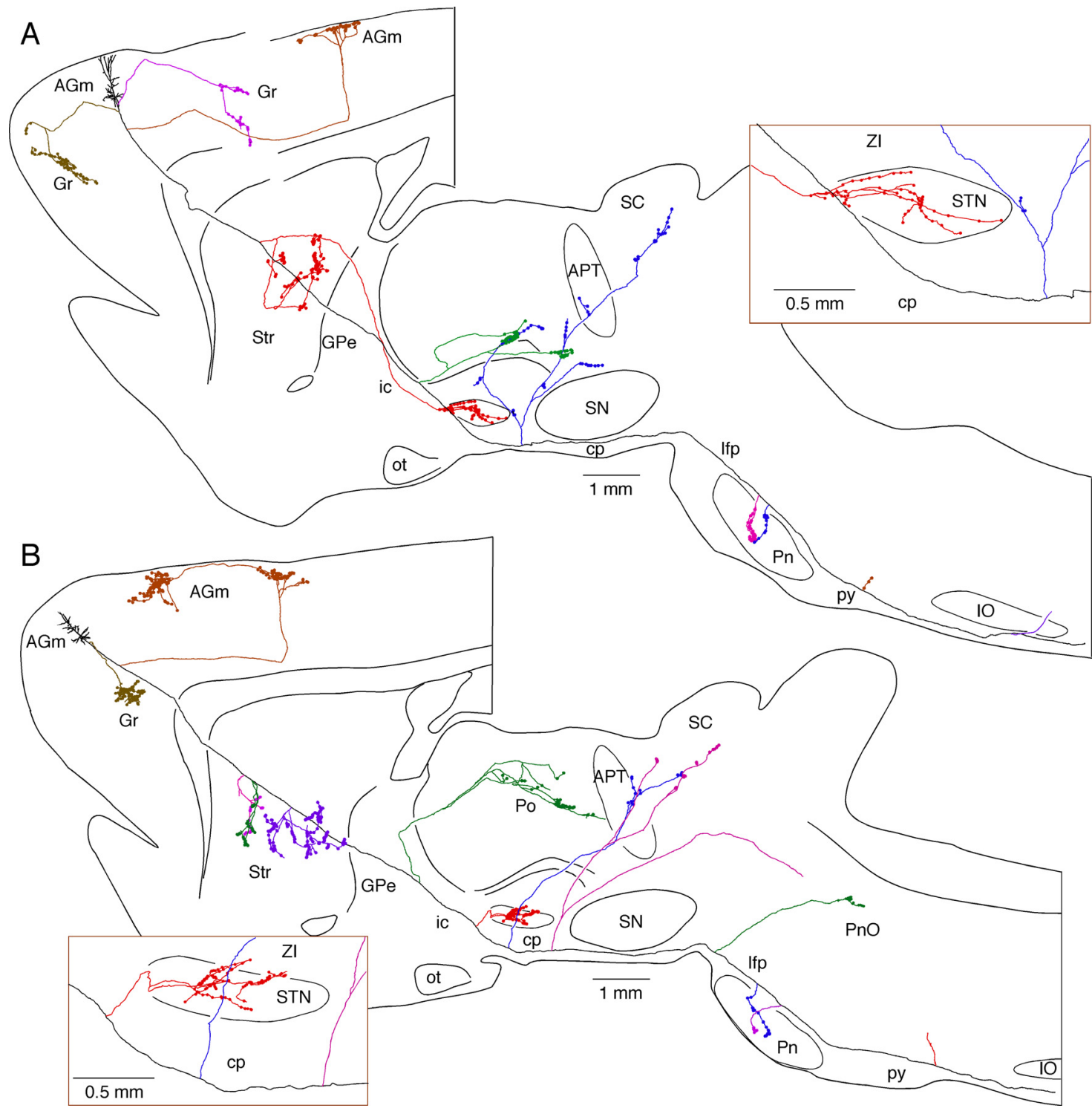

Figure 3. Axons of two AGm pyramidal tract neurons that emit multiple collaterals including STN, Str, thalamic, and pontine nuclei. Both neurons shown in $\boldsymbol{A}$ and $\boldsymbol{B}$ had cortical collaterals innervating AGm, Gr, and Str. The thalamic collateral of the neuron in $A$ traveled through the ventral part of the thalamus while that of in $B$ traveled through the middle of the thalamus. One of the cerebral peduncle collaterals of the neuron in $\boldsymbol{B}$ traversed STN and then to Zl without forming boutons. GPe, Globus pallidus external segment; Gr, granular cortex; ic, internal capsule; I0, inferior olive; ot, optic tract; cp, cerebral peduncle; Ifp, longitudinal fasciculus of the pons; py, medullary pyramid.

ons (mean 96.8; range 25-204; $n=4$ ) than those of AGl axons (mean 39.1; range $14-68 ; n=9$ ) (Figs. 2, 3) (no statistical analysis was performed because of potential incomplete tracings). The areas innervated by the collaterals were located lateral or ventrolateral to the parent axon. In one neuron, a thin collateral that emerged in Str innervated Str and then descended to STN. No Str collateral was found on the remaining 12 of 25 neurons.

Thalamic and mesencephalic collaterals

The parent axons of all 25 neurons passed through the external segment of the globus pallidus without emitting collaterals and then entered the internal capsule. Nineteen of the 25 axons emitted a collateral in the internal capsule (called internal capsule collaterals hereafter). These internal capsule collaterals took two separate courses to innervate the thalamus. Thirteen of 19 collaterals traversed the reticular thalamic nucleus and the ventrolateral thalamic nucleus and formed sparse terminal fields in the posterior thalamic nuclear group (Figs. 4C, 7C). Three of these 13 axons ran further caudally and innervated the intralaminar nuclei (Figs. 2A, 3B). The remaining 6 of the 19 internal capsule collaterals ran within the medial lemniscus or slightly dorsally within the ventral posteromedial thalamic nucleus, and reached and formed a terminal field in the posterior part of the posterior thalamic nucleus (Po). Two of these ran further caudally to the deep mesencephalic nuclei (DpMe) (Figs. 2B, 3A). None of these thalamic collaterals formed terminal fields in the reticular thalamic nucleus.

The parent axons of most of the cortico-STN neurons (23 of 25) also emitted one to four collaterals innervating the thalamus and mesencephalon at the level between the caudal part of STN and the rostral part of the substantia nigra $(\mathrm{SN})$. These collaterals are referred to hereafter as the cerebral peduncle collaterals. The parent axon of a nonpyramidal tract cortico-STN neuron ran dorsally with other cerebral peduncle collaterals and innervated to the ventral part of the anterior pretectal nucleus (APT) and 

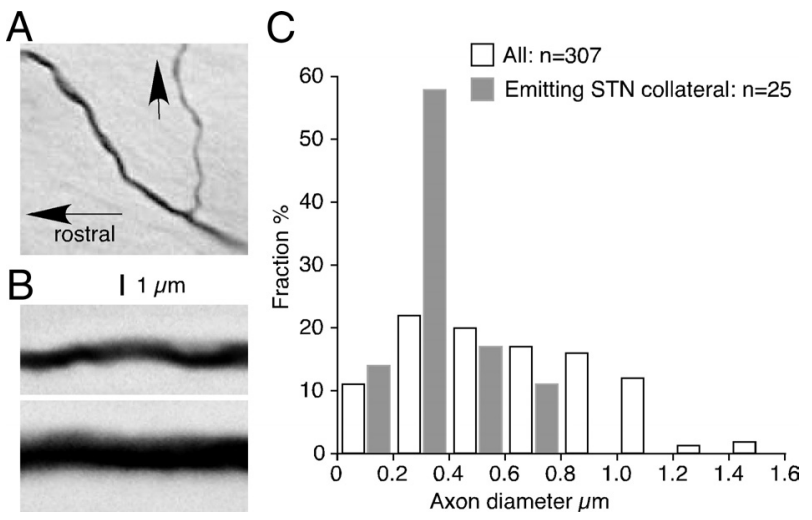

Figure 4. $\quad \boldsymbol{A}, \boldsymbol{B}$, Photomontages of BDA-labeled axons with ( $\boldsymbol{A}$, arrow) and without $(\boldsymbol{B})$ emitting STN collateral. The orientations of the axons in $\boldsymbol{B}$ were artificially aligned horizontally. The calibration in $\boldsymbol{A}$ also applies to $\boldsymbol{B}$. C, Distributions of diameters of parent axons in the cerebral peduncle at the level of STN. The diameter of a thin smooth portion, between varicosities, of each axon was observed using a $100 \times$ oil objective and was assigned to one of $0.2 \mu \mathrm{m}$ increment categories.

deep layers of SC, as described earlier. Some of the cerebral peduncle collaterals passed dorsomedially through STN and SN without forming boutons, innervated into the ZI, the caudal part of Po, the ventral part of APT, and the deep layers of SC. Other innervation sites were the DpMe and the pedunculopontine tegmentum (PPTg). Thus, all of the cortico-STN neurons innervated the thalamus and mesencephalon, most of them through both internal capsule collaterals and through more caudal cerebral peduncle collaterals (Table 1).

\section{Pontine nucleus collaterals}

All of the 24 parent axons traveling in the longitudinal fasciculus of the pons emitted one or two collaterals in Pn, and a parent axon terminated in Pn, as described earlier. In Pn, thin collaterals bearing en passant boutons formed small but dense terminal fields that overlapped with those formed by other axon collaterals of different neurons (Fig. 7E). Because of the dense and complicated axonal fields, only a part of the collaterals was traced.

\section{Pontine and medullary reticular nucleus}

Eleven of the 23 parent axons descending through the longitudinal fasciculus and 11 of the 14 axons descending through the pyramidal tract emitted collaterals to innervate the pontine and medullary reticular nuclei. None of the parent or collateral axons was traced to cranial motor nuclei.

\section{Axonal morphology}

The morphological features of axons at various terminal sites were similar. In general, long secondary or higher-order branches took tortuous courses and bore sparse en passant and occasional pedunculated boutons with varying intervals, 5-20 $\mu \mathrm{m}$ on long axons and $2-5 \mu \mathrm{m}$ at terminal portions of axons (Figs. 1, 7). The terminal density in STN, Str, thalamic nuclei, and SC were sparse, while layer I of the cortex and Pn contained denser axonal branches and terminals in smaller terminal fields (Fig. $7 B, E$ ). These morphological features are similar to previously described Str and thalamic collaterals of motor cortex layer $\mathrm{V}$ pyramidal tract axons (Cowan and Wilson, 1994; Deschênes et al., 1994; Parent and Parent, 2006).

\section{Discussion}

\section{Neuronal type}

This study revealed that the powerful cortico-STN inputs described in previous studies were achieved by a small fraction of long-range corticofugal neurons that emit collaterals not only to STN but also to other brain areas. The pyramidal tract neurons in the motor cortex are heterogeneous groups of cells having different morphological and physiological properties. The somatic location and somatodendritic morphology of cortico-STN neurons resemble those of the agranular cortex layer $\mathrm{V}$ cells projecting to subcortical regions, including the intralaminar and posteromedial thalamic nucleus, SC, pontine nuclei, and medullary reticular formation (Tseng and Prince, 1993; Deschênes et al., 1994; Veinante and Deschênes, 2003; Morishima et al., 2011). Paz et al. (2005) revealed that cortical neurons responding with antidromic spikes to STN stimulation had a prominent hyperpolarizing sag and rebound depolarization and generated rhythmic burst discharges synchronized with electrocorticogram spikes. These physiological properties resemble those of a subgroup of rat corticospinal neurons characterized in vitro that have larger somatic sizes and are capable of generating repetitive burst spikes (Tseng and Prince, 1993; Wang and McCormick, 1993). These, together with the present observations, suggest that cortico-STN neurons are large pyramidal neurons with relatively spine-free dendrites that are capable of generating burst firings. However, whether the cortico-STN neurons have unique morphological and physiological features distinguishable from other subpopulations is unknown at this time.

The present BDA injection sites were in the orofacial and vibrissa motor areas, according to intracranial microstimulation studies (Hall and Lindholm, 1974; Donoghue and Wise, 1982; Brecht et al., 2004). These areas project to both the bulbar motor nuclei and the cervical spinal cord (Donoghue et al., 1979; Nudo and Masterton, 1990; Grinevich et al., 2005). In this study, we identified about one third of cortico-STN neurons as being corticospinal neurons, while none were identified as corticobulbar neurons. Although it is possible that some of the axons that faded out in the medullary pyramidal tract (i.e., 10 of 25 axons) projected to the bulbar motor nuclei, another possibility is that cortico-STN neurons are involved in associative sensorimotor control of other body parts but not in the direct control of orofacial muscle movement.

\section{Subthalamic collaterals}

The STN collaterals arose from thin-to-medium-sized parent axons but not from thick axons. This observation coincides with the conduction velocity of the cortico-STN axons $(5 \mathrm{~m} / \mathrm{s})$ estimated from antidromic latency data (Paz et al., 2005), which is on the slow side of the conduction velocity $(5-19 \mathrm{~m} / \mathrm{s})$ of corticospinal axons in rats (Mediratta and Nicoll, 1983). However, the latency of the cortico-STN orthodromic response is relatively short, 3-5 ms (Kitai and Deniau, 1981; Fujimoto and Kita, 1993; Degos et al., 2008), due to the unique membrane properties of STN neurons that enable the excitatory inputs to trigger action potentials with very short delays (Farries et al., 2010).

STN collaterals formed sparse terminal fields of various sizes in the motor territory of STN. We found that AGm axons tend to have larger terminal fields with more boutons than AGl axons. However, this may not reflect overall innervation densities because the fraction of STN-projecting neurons in AGm was lower than in AGl. Cortical axons form synapses predominantly with spins and small-diameter dendrites of STN neurons (Moriizumi et al., 1987; Bevan et al., 1995). The size of the dendritic arbor of STN neurons also varies considerably among neurons (Yelnik and Percheron, 1979; Kita et al., 1983; Pearson et al., 1985). Although how each cortical axon selects target neurons in STN is unknown, it is likely that each cortical neuron targets a small fraction of STN neurons and that each STN receives inputs from 
Table 1. Summary of the tracing results of $18 \mathrm{AGI}$ and $7 \mathrm{AGm}$ axons emitting STN collaterals shows origins and final tracing sites of the main axons and other collateral sites

\begin{tabular}{|c|c|c|c|c|c|c|c|c|}
\hline & Traced from-to & $C x$ & Str & ic-Th & cp-Th/Me & $\mathrm{Pn}$ & Pontine reticular & Medulla reticular \\
\hline AGI-1 & Soma-py & ++ & ++ & + & + & + & - & $?$ \\
\hline AGI-2 & Soma-des & ++ & + & + & + & ++ & - & - \\
\hline AGI-3 & Soma-py & ++ & - & - & + & + & + & $?$ \\
\hline AGI-4 & Soma-dcs & + & ++ & + & + & ++ & - & + \\
\hline $\mathrm{AGI}-5$ & Soma-SC & + & ++ & + & + & NA & NA & NA \\
\hline AGI-6 & Soma-py & + & + & + & ++ & + & - & $?$ \\
\hline AGI-7 & Soma-dcs & + & - & + & ++ & + & - & + \\
\hline AGI-8 & Soma-des & - & + & + & +++ & ++ & +++ & + \\
\hline AGI-9 & Soma-py & - & - & + & + & + & + & $?$ \\
\hline AGI-10 & Soma-py & - & - & + & + & ++ & + & $?$ \\
\hline AGI-11 & Soma-dcs & - & - & + & + & ++ & ++ & - \\
\hline AGI-12 & Soma-dcs & - & - & + & - & + & +++ & ++ \\
\hline AGI-13 & ?-py & + & + & - & + & + & - & $?$ \\
\hline AGI-14 & ?-py & $?$ & ++ & + & + & + & - & $?$ \\
\hline AGI-15 & ?-pyd & $?$ & - & ++ & + & + & - & ++ \\
\hline AGl-16 & ?-Pn & $?$ & - & + & ++ & + & NA & NA \\
\hline AGI-17 & ?-pyd & $?$ & - & - & + & + & - & - \\
\hline AGI-18 & ?-py & $?$ & + & - & + & ++ & + & $?$ \\
\hline Subtotal & $12 / 18$ & $8 / 13$ & $9 / 18$ & $14 / 18$ & $17 / 18$ & $17 / 17$ & $7 / 16$ & $6 / 9$ \\
\hline AGm-1 & Soma-py & ++ & ++ & + & + & ++ & ++ & $?$ \\
\hline$A G m-2$ & Soma-dcs & - & ++ & + & + & ++ & + & + \\
\hline $\mathrm{AGm}-3$ & Soma-dcs & - & ++ & + & ++ & + & + & ++ \\
\hline AGm-4 & Soma-pyd & +++ & + & + & + & ++ & - & + \\
\hline$A G m-5$ & Soma-py & - & - & + & ++ & ++ & - & $?$ \\
\hline AGm-6 & ?-pyd & + & - & - & + & + & + & + \\
\hline AGm-7 & ?-pyd & + & - & - & + & + & + & + \\
\hline Subtotal & $5 / 7$ & $4 / 7$ & $4 / 7$ & $5 / 7$ & $7 / 7$ & $7 / 7$ & $5 / 7$ & $5 / 5$ \\
\hline Total & $17 / 25$ & $12 / 20$ & $13 / 25$ & $19 / 25$ & $24 / 25$ & $24 / 24$ & $12 / 23$ & $11 / 14$ \\
\hline
\end{tabular}

+ , Number of identified collaterals; - , no collateral; ?, unknown; NA, not applicable; cp-Th/Me, cerebral peduncle-thalamus and mesencephalon; C $x$, cortex; dcs, dorsal corticospinal tract; ic-Th, internal capsule-thalamic; py, medullary pyramid; pyd, pyramidal decussation; Th, thalamus.

many cortical neurons, possibly from both AGl and AGm, based on the present single axonal morphology, the dendritic morphology, and the total number of neurons in rat STN (Oorschot, 1996).

\section{Other common innervation sites}

Cortex and striatum

The axons of pyramidal tract neurons emit cortical and Str collaterals (Donoghue and Kitai, 1981; Cowan and Wilson, 1994; Reiner et al., 2003; Veinante and Deschênes, 2003; Parent and Parent, 2006). In this study, intracortical collaterals were found in 12 neurons. Some of the neurons had collaterals innervating to both agranular and granular cortices. The trajectory and the terminal sites of collaterals resembled those of layer $\mathrm{V}$ corticofugal neurons in the rat motor cortex having collaterals projecting to sensory barrel field as described by Veinante and Deschênes (2003). We did not find any cortical collateral in 8 of the 17 axons traced to their somata. This is an intriguing observation because there have been no similar reports. We found Str collaterals in only 12 of 25 neurons, and there was a tendency for those neurons having cortical collaterals to also have Str collaterals. We think that these observations are not related to the staining intensity of individual neurons because the neurons without cortical or Str collaterals

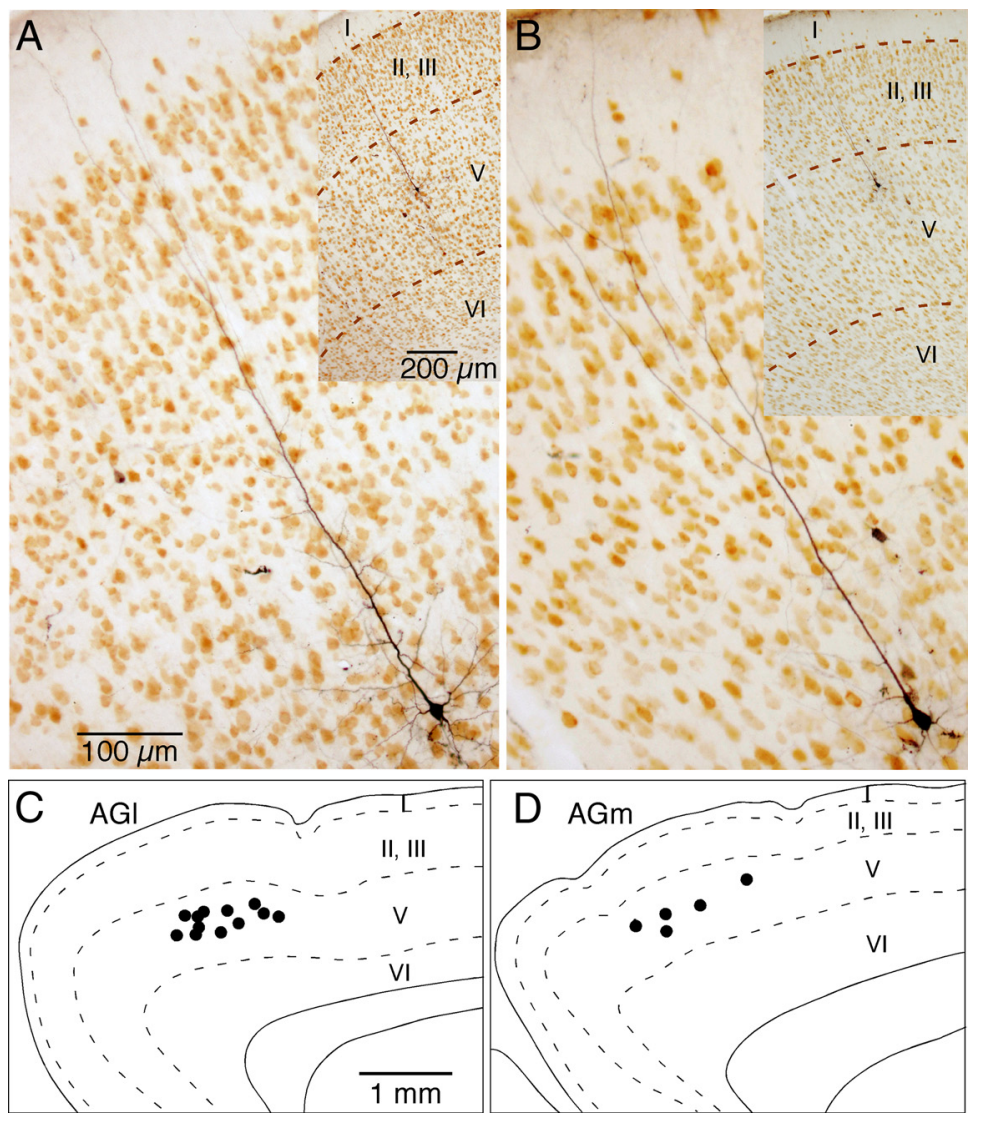

Figure 5. $\quad A, B$, Photomicrographs of STN-innervating neurons in NeuN-stained sections. $C, D$, Approximate locations of somata of STN-innervating neurons in AGI and AGm. Most of the somata were located in the upper-middle part of layer V. 


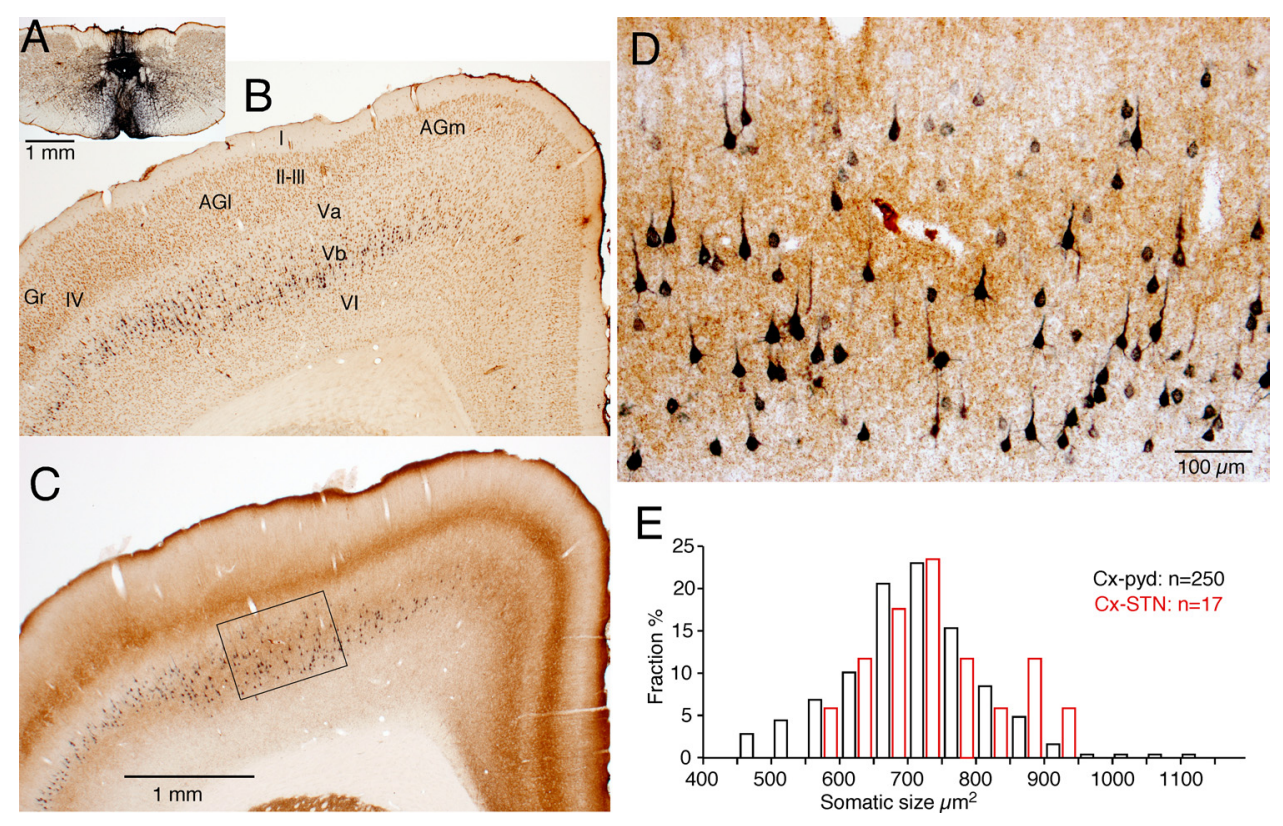

Figure 6. Pyramidal tract neurons labeled after FG injection into the pyramidal decussation. $A$, FG injection site. $\boldsymbol{B}, A$ coronal section double labeled for $F G$ and NeuN. $\boldsymbol{C}$, An adjacent section shown in $\boldsymbol{B}$ was double labeled for FG and VGLUT2. FG-labeled neurons in AGl and AGm are located mostly in the VGLUT2-rich layer Vb, while those in Gr are mostly in the VGLUT2-poor layer Va. $\boldsymbol{D}$, A high-magnification view of the area marked in C. Small to large FG neurons appear to be randomly distributed in the layer Vb. $\boldsymbol{E}$, Somatic size (i.e., shadow area) of FG-labeled and STN-innervating neurons. Gr, Granular cortex; pyd, pyramidal decussation.

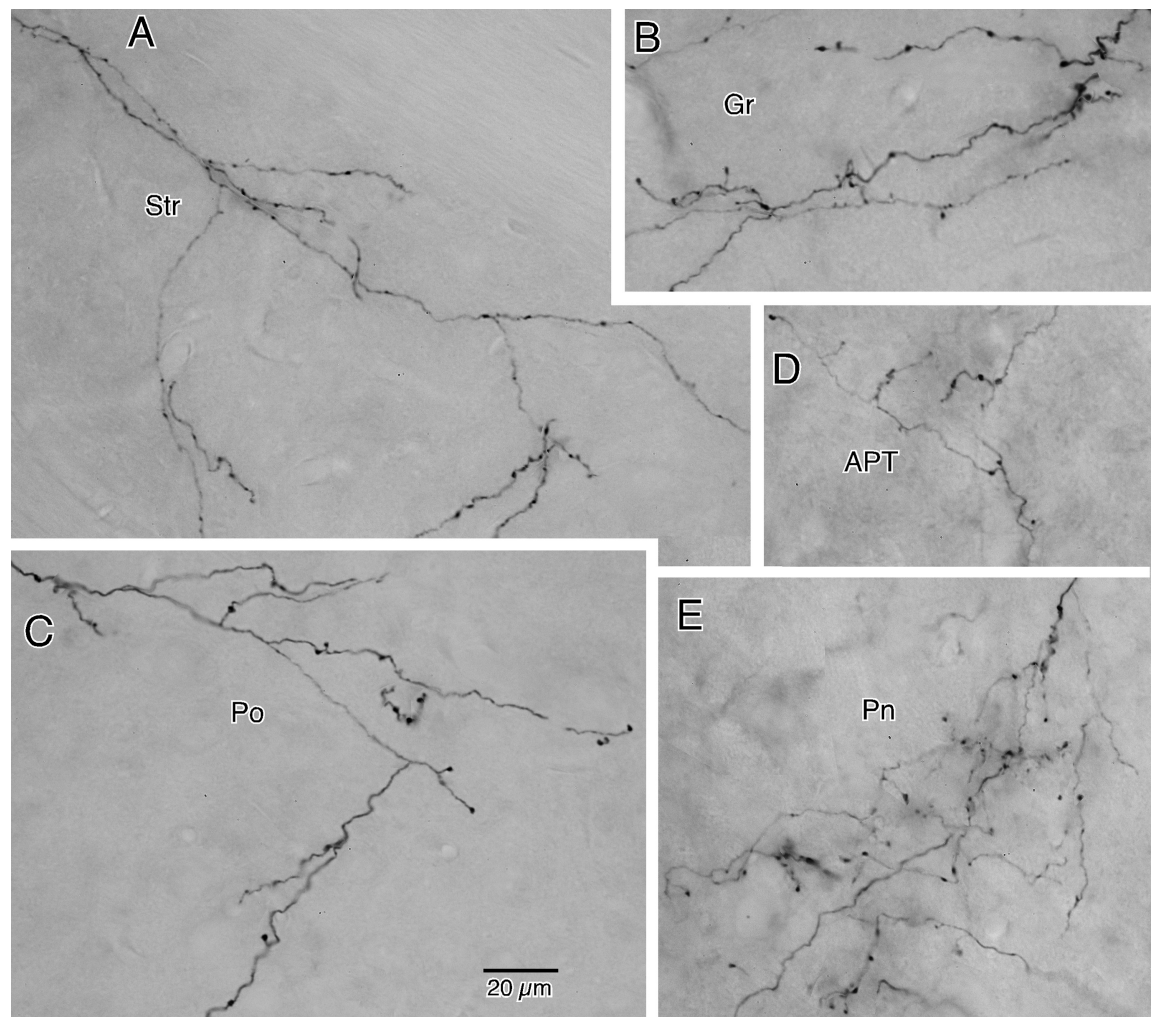

Figure 7. $\quad A-E$, Photomontages of BDA-labeled axon collaterals in Str $(A)$, layer l of granular cortex $(G r ; B), P o(C), A P T(D)$, and $\operatorname{Pn}(\boldsymbol{E})$. Axons in $\boldsymbol{B}$ and $\boldsymbol{E}$ include those that originated from multiple neurons. Collateral axons at various terminal sites had very similar morphological features in that long secondary or higher-order branches took tortuous courses and bore sparse en passant and occasional pedunculated boutons at various intervals. The calibration in Capplies to all photomontages.

often emitted collaterals in the pons and medulla. The results suggest that some pyramidal tract neurons innervate other cortical areas, Str, and STN, and that other STN-projecting pyramidal tract neurons actually do not emit cortical and Str collateral axons in rats, although these observations could be due to other technical limitations.

\section{In the thalamus and midbrain}

This study revealed that cortico-STN neurons innervate multiple sites in the thalamus and brainstem. Féger et al. (1994) also reported that a small number of cortical neurons were doubly labeled after retrograde tracer injections into STN and parafascicular nucleus. Most of corticoSTN neurons emitted collaterals innervating the thalamus at the level of the internal capsule. As described by Deschênes et al. (1994), some of the long-range corticofugal axons emit thalamic collaterals, which do not emit branches innervating the reticular thalamic nucleus but arborize within associative and intralaminar thalamic nuclei. This study revealed that most of the cortico-STN neurons also emit cerebral peduncle collaterals that innervate ZI, posterior thalamic nuclei, SC, and/or PPTg. Innervations from the motor cortex to these areas have been known to occur (Shammah-Lagnado et al., 1985; Tseng and Prince, 1993; Mitrofanis and Mikuletic, 1999; Veinante and Deschênes, 2003; Parent and Parent, 2006). This study shows that most of the cortico-STN neurons emit multiple collaterals to innervate these brain areas.

An unexpected innervation site in the diencephalon was ZI. Some axon collaterals innervating STN traveled further cau- 
dodorsally and innervated ZI. The cerebral peduncle collaterals of some cortico-STN neurons also innervated ZI, as mentioned above. The results suggest that more than half of cortico-STN neurons innervate ZI in rats. It has been reported that 6-hydroxydopamine lesion of the mesencephalic dopaminergic neurons increased neuronal activity of ZI in rats (Périer et al., 2000). DBS of the caudal ZI is effective for the treatment of akinesia and bradykinesia in Parkinson's patients (Henderson et al., 2002; Voges et al., 2002) and intractable proximal tremor (Murata et al., 2003; Plaha et al., 2006). These reports, together with our present observations, suggest that STN and caudal ZI receive common inputs from the motor cortex, that the caudal $\mathrm{ZI}$ is involved in associative motor control (Mitrofanis 2005), and that the DBS of caudal ZI might activate neurons and axons at the stimulus site, including the cortico-STN axons and neurons postsynaptic to the axons.

\section{In the pons}

We found that all of the parent axons of cortico-STN neurons descending through the longitudinal fasciculus emitted one or two collaterals in Pn, including a parent axon that terminated in Pn. These observations suggest that the basal ganglia and the cerebellum may receive the same information from the motor cortex through Str, STN, and Pn.

\section{Functional implication}

This study revealed that the cortico-STN projection is formed by collaterals of a small subpopulation of small-to-medium-sized long-range corticofugal axons, which also emit collaterals innervating multiple other brain sites. These innervation sites have different functional modality at different levels, including the motor and sensory functions in the telencephalon; associative and sensory integration in posterior thalamic nuclei and in the mesopontine area; reticular activation systems in the lower brainstem; and probably modulation of spinal functions through slow conducting pyramidal tract axons. Having small-diameter parent axons and diverse innervation sites suggest that those cortical neurons are suitable for association or coordination of multiple brain areas for performing complex tasks but not for rapidly sending efferent copies of rapid-firing movement commands, which require large caliber axons (Perge et al., 2012). It is also conceivable that STN itself is involved in an associative function by integrating various modes of inputs through globus pallidus external segment, intralaminar thalamic nuclei, SC, and PPTg that are required to perform complex behaviors by integrating internal and external inputs. This assumption is supported by the observation that STN is more active during complex than simple movement tasks (Lehéricy et al., 2006). The present results also suggest that activation of cortico-STN axons during DBS of the subthalamus may activate all the brain sites that are innervated by the multiple collaterals of cortico-STN neurons and that the activation of various motor association areas may contribute to the manifestation of DBS effects.

\section{References}

Albin RL, Young AB, Penney JB (1989) The functional anatomy of basal ganglia disorders. Trends Neurosci 12:366 -375 .

Bevan MD, Francis CM, Bolam JP (1995) The glutamate-enriched cortical and thalamic input to neurons in the subthalamic nucleus of the rat: convergence with GABA-positive terminals. J Comp Neurol 361:491-511.

Brecht M, Krauss A, Muhammad S, Sinai-Esfahani L, Bellanca S, Margrie TW (2004) Organization of rat vibrissa motor cortex and adjacent areas according to cytoarchitectonics, microstimulation, and intracellular stimulation of identified cells. J Comp Neurol 479:360-373.
Canteras NS, Shammah-Lagnado SJ, Silva BA, Ricardo JA (1990) Afferent connections of the subthalamic nucleus: a combined retrograde and anterograde horseradish peroxidase study in the rat. Brain Res 513:43-59.

Chang HT, Kuo H, Whittaker JA, Cooper NG (1990) Light and electron microscopic analysis of projection neurons retrogradely labeled with Fluoro-Gold: notes on the application of antibodies to Fluoro-Gold. J Neurosci Methods 35:31-37.

Cowan RL, Wilson CJ (1994) Spontaneous firing patterns and axonal projections of single corticostriatal neurons in the rat medial agranular cortex. J Neurophysiol 71:17-32.

Degos B, Deniau JM, Le Cam J, Mailly P, Maurice N (2008) Evidence for a direct subthalamo-cortical loop circuit in the rat. Eur J Neurosci 27:2599-2610.

DeLong MR (1990) Primate models of movement disorders of basal ganglia origin. Trends Neurosci 13:281-285.

Deschênes M, Bourassa J, Pinault D (1994) Corticothalamic projections from layer $\mathrm{V}$ cells in rat are collaterals of long-range corticofugal axons. Brain Res 664:215-219.

Donoghue JP, Kitai ST (1981) A collateral pathway to the neostriatum from corticofugal neurons of the rat sensory-motor cortex: an intracellular HRP study. J Comp Neurol 201:1-13.

Donoghue JP, Wise SP (1982) The motor cortex of the rat: cytoarchitecture and microstimulation mapping. J Comp Neurol 212:76-88.

Donoghue JP, Kerman KL, Ebner FF (1979) Evidence for two organizational plans within the somatic sensory-motor cortex of the rat. J Comp Neurol 183:647-663.

Farries MA, Kita H, Wilson CJ (2010) Dynamic spike threshold and zero membrane slope conductance shape the response of subthalamic neurons to cortical input. J Neurosci 30:13180-13191.

Féger J, Bevan M, Crossman AR (1994) The projections from the parafascicular thalamic nucleus to the subthalamic nucleus and the striatum arise from separate neuronal populations: a comparison with the corticostriatal and corticosubthalamic efferents in a retrograde fluorescent doublelabelling study. Neuroscience 60:125-132.

Fujimoto K, Kita H (1993) Response characteristics of subthalamic neurons to the stimulation of the sensorimotor cortex in the rat. Brain Res 609:185-192.

Giuffrida R, Li Volsi G, Maugeri G, Perciavalle V (1985) Influences of pyramidal tract on the subthalamic nucleus in the cat. Neurosci Lett 54:231-235

Graybiel AM, Aosaki T, Flaherty AW, Kimura M (1994) The basal ganglia and adaptive motor control. Science 265:1826-1831.

Grinevich V, Brecht M, Osten P (2005) Monosynaptic pathway from rat vibrissa motor cortex to facial motor neurons revealed by lentivirus-based axonal tracing. J Neurosci 25:8250-8258.

Hall RD, Lindholm EP (1974) Organization of motor and somatosensory neocortex of the albino rat. Brain Res 66:23-38.

Henderson JM, Pell M, O'Sullivan DJ, McCusker EA, Fung VS, Hedges P, Halliday GM (2002) Postmortem analysis of bilateral subthalamic electrode implants in Parkinson's disease. Mov Disord 17:133-137.

Hornykiewicz O (2006) The discovery of dopamine deficiency in the parkinsonian brain. J Neural Transm Suppl (70):9-15.

Israel Z, Bergman H (2008) Pathophysiology of the basal ganglia and movement disorders: from animal models to human clinical applications. Neurosci Biobehav Rev 32:367-377.

Iwahori N (1978) A Golgi study on the subthalamic nucleus of the cat. J Comp Neurol 182:383-397.

Kita H (1994) Physiology of two disynaptic pathways from the sensorimotor cortex to the basal ganglia output nuclei. In: The basal ganglia IV (Percheron G, McKenzie JS, Féger J, eds), pp 263-276, New York: Plenum.

Kita H, Chang HT, Kitai ST (1983) The morphology of intracellularly labeled rat subthalamic neurons: a light microscopic analysis. J Comp Neurol 215:245-257.

Kitai ST, Deniau JM (1981) Cortical inputs to the subthalamus: intracellular analysis. Brain Res 214:411-415.

Kitai ST, Kita H (1987) Anatomy and physiology of the subthalamic nucleus: a driving force of the basal ganglis. New York: Plenum.

Kolomiets BP, Deniau JM, Mailly P, Ménétrey A, Glowinski J, Thierry AM (2001) Segregation and convergence of information flow through the cortico- subthalamic pathways. J Neurosci 21:5764-5772.

Kubota Y, Hatada S, Kondo S, Karube F, Kawaguchi Y (2007) Neocortical 
inhibitory terminals innervate dendritic spines targeted by thalamocortical afferents. J Neurosci 27:1139-1150.

Künzle H (1978) An autoradiographic analysis of the efferent connections from premotor and adjacent prefrontal regions (areas 6 and 9) in macaca fascicularis. Brain Behav Evol 15:185-234.

Lehéricy S, Bardinet E, Tremblay L, Van de Moortele PF, Pochon JB, Dormont D, Kim DS, Yelnik J, Ugurbil K (2006) Motor control in basal ganglia circuits using fMRI and brain atlas approaches. Cereb Cortex 16:149-161.

Mediratta NK, Nicoll JA (1983) Conduction velocities of corticospinal axons in the rat studied by recording cortical antidromic responses. J Physiol 336:545-561.

Mitrofanis J (2005) Some certainty for the "zone of uncertainty"? Exploring the function of the zona incerta. Neuroscience 130:1-15.

Mitrofanis J, Mikuletic L (1999) Organisation of the cortical projection to the zona incerta of the thalamus. J Comp Neurol 412:173-185.

Monakow KH, Akert K, Künzle H (1979) Projections of precentral and premotor cortex to the red nucleus and other midbrain areas in Macaca fascicularis. Exp Brain Res 34:91-105.

Moriizumi T, Nakamura Y, Kitao Y, Kudo M (1987) Ultrastructural analyses of afferent terminals in the subthalamic nucleus of the cat with a combined degeneration and horseradish peroxidase tracing method. J Comp Neurol 265:159-174.

Morishima M, Morita K, Kubota Y, Kawaguchi Y (2011) Highly differentiated projection-specific cortical subnetworks. J Neurosci 31:10380-10391.

Murata J, Kitagawa M, Uesugi H, Saito H, Iwasaki Y, Kikuchi S, Tashiro K, Sawamura Y (2003) Electrical stimulation of the posterior subthalamic area for the treatment of intractable proximal tremor. J Neurosurg 99:708-715.

Nambu A, Tokuno H, Hamada I, Kita H, Imanishi M, Akazawa T, Ikeuchi Y, Hasegawa N (2000) Excitatory cortical inputs to pallidal neurons via the subthalamic nucleus in the monkey. J Neurophysiol 84:289-300.

Nudo RJ, Masterton RB (1990) Descending pathways to the spinal cord, III: Sites of origin of the corticospinal tract. J Comp Neurol 296:559-583.

Oorschot DE (1996) Total number of neurons in the neostriatal, pallidal, subthalamic, and substantia nigral nuclei of the rat basal ganglia: a stereological study using the cavalieri and optical disector methods. J Comp Neurol 366:580-599.

Orieux G, François C, Féger J, Hirsch EC (2002) Consequences of dopami- nergic denervation on the metabolic activity of the cortical neurons projecting to the subthalamic nucleus in the rat. J Neurosci 22:8762-8770.

Parent M, Parent A (2006) Single-axon tracing study of corticostriatal projections arising from primary motor cortex in primates. J Comp Neurol 496:202-213.

Paz JT, Deniau JM, Charpier S (2005) Rhythmic bursting in the corticosubthalamo-pallidal network during spontaneous genetically determined spike and wave discharges. J Neurosci 25:2092-2101.

Pearson JC, Norris JR, Phelps CH (1985) Subclassification of neurons in the subthalamic nucleus of the lesser bushbaby (Galago senegalensis): a quantitative Golgi study using principal components analysis. J Comp Neurol 238:323-339.

Perge JA, Niven JE, Mugnaini E, Balasubramanian V, Sterling P (2012) Why do axons differ in caliber? J Neurosci 32:626-638.

Périer C, Vila M, Féger J, Agid Y, Hirsch EC (2000) Functional activity of zona incerta neurons is altered after nigrostriatal denervation in hemiparkinsonian rats. Exp Neurol 162:215-224.

Plaha P, Ben-Shlomo Y, Patel NK, Gill SS (2006) Stimulation of the caudal zona incerta is superior to stimulation of the subthalamic nucleus in improving contralateral parkinsonism. Brain 129:1732-1747.

Reiner A, Jiao Y, Del Mar N, Laverghetta AV, Lei WL (2003) Differential morphology of pyramidal tract-type and intratelencephalically projecting-type corticostriatal neurons and their intrastriatal terminals in rats. J Comp Neurol 457:420-440.

Shammah-Lagnado SJ, Negrão N, Ricardo JA (1985) Afferent connections of the zona incerta: a horseradish peroxidase study in the rat. Neuroscience 15:109-134.

Tseng GF, Prince DA (1993) Heterogeneity of rat corticospinal neurons. J Comp Neurol 335:92-108.

Veinante P, Deschênes M (2003) Single-cell study of motor cortex projections to the barrel field in rats. J Comp Neurol 464:98-103.

Voges J, Volkmann J, Allert N, Lehrke R, Koulousakis A, Freund HJ, Sturm V (2002) Bilateral high-frequency stimulation in the subthalamic nucleus for the treatment of Parkinson disease: correlation of therapeutic effect with anatomical electrode position. J Neurosurg 96:269-279.

Wang Z, McCormick DA (1993) Control of firing mode of corticotectal and corticopontine layer $\mathrm{V}$ burst-generating neurons by norepinephrine, acetylcholine, and 1S,3R-ACPD. J Neurosci 13:2199-2216.

Yelnik J, Percheron G (1979) Subthalamic neurons in primates: a quantitative and comparative analysis. Neuroscience 4:1717-1743. 